\title{
Atomic structure of amorphous and crystallized $\mathrm{Ge}_{15} \mathrm{Sb}_{85}$
}

\author{
Peter Zalden, ${ }^{1}$ Christophe Bichara, ${ }^{2}$ Julia van Eijk, ${ }^{1}$ Carolin Braun, ${ }^{3}$ \\ Wolfgang Bensch, ${ }^{3}$ and Matthias Wuttig ${ }^{1, a)}$ \\ ${ }^{1}$ I. Physikalisches Institut (IA), RWTH Aachen, 52056 Aachen, Germany \\ ${ }^{2}$ CiNaM-Centre Interdisciplinaire de Nanoscience de Marseille, Campus de Luminy, \\ 13288 Marseille, France \\ ${ }^{3}$ Institut für Anorganische Chemie, Universität Kiel, Max-Eyth Str. 2, 24118 Kiel, Germany
}

(Received 9 November 2009; accepted 8 March 2010; published online 21 May 2010)

\begin{abstract}
$\mathrm{Ge}_{15} \mathrm{Sb}_{85}$ is a promising material for phase-change memory applications owing to its very short crystallization times. As deposited amorphous samples of sputter deposited $\mathrm{Ge}_{15} \mathrm{Sb}_{85}$ have been investigated by extended x-ray absorption fine structure (EXAFS) measurements on both, $\mathrm{Sb}$ and Ge $K$ absorption edges. After crystallizing the specimen, x-ray diffraction (XRD) and EXAFS measurements have been performed to analyze the atomic structure at different annealing conditions. Thus, experimental techniques focusing on the long range order as well as on the local order have been combined. Sb atoms have on average 3.2(2) nearest neighbors, while Ge atoms have 4.0(3). The Ge-Ge and $\mathrm{Ge}-\mathrm{Sb}$ bond lengths are determined to 2.46(2) and 2.66(1) $\AA$, respectively and agree well with those observed in the amorphous phase of the common phase-change material $\mathrm{Ge}_{2} \mathrm{Sb}_{2} \mathrm{Te}_{5}$. After crystallizing the sample at $250{ }^{\circ} \mathrm{C}$, very different EXAFS spectra with modified $\mathrm{Ge}-\mathrm{Sb}$ bond lengths are observed. The higher concentration of Ge neighbors at the Ge edge as compared to the as-deposited sample is indicative for phase separation. For the corresponding sample, XRD does not show reflections of Ge, which indicates that the agglomeration of $\mathrm{Ge}$ is amorphous or below the coherence length of the x-radiation. The EXAFS spectrum shows a superposition of two phases: one with bond lengths which agree with $s p^{3}$-hybridized Ge [2.43(1) $\AA]$ and another one with longer Ge-Ge bond lengths [2.79(8) $\AA$ ]. This result can be explained by phase separation in the material. () 2010 American Institute of Physics. [doi:10.1063/1.3380667]
\end{abstract}

\section{INTRODUCTION}

Phase-change memory (PCM) devices are promising candidates for future nonvolatile data storage, ${ }^{1,2}$ because they can be switched faster than commonly employed flash memory. ${ }^{3,4}$ At the same time, the scaling potential, i.e., the maximum achievable storage density of phase-change cells is expected to surpass flash memory. ${ }^{3}$ The phase-change mechanism is based on the different electronic resistivity of the amorphous (glassy) and crystalline state. ${ }^{5}$ Sb-based alloys were reported to exhibit attractive properties for PCM applications. ${ }^{6-8}$ The speed of these devices is limited by the time necessary to fully crystallize the active material. Phase transformations induced by short laser pulses have shown that full crystallization can take place within 0.4 ns (Ref. 9) in the eutectic $\mathrm{Ge}-\mathrm{Sb}$ binary system, i.e., $\mathrm{Ge}_{15} \mathrm{Sb}_{85}$, with $\mathrm{Ge}$ added solely to prevent "explosive crystallization"10 already at room temperature. The addition of Ge stabilizes the amorphous structure and increases the crystallization temperature. ${ }^{11,12}$ The crystallization temperature of $\mathrm{Ge}_{15} \mathrm{Sb}_{85}$ $(512 \mathrm{~K})$ is higher than that of common phase change materials like $\mathrm{Ge}_{2} \mathrm{Sb}_{2} \mathrm{Te}_{5}(446 \mathrm{~K}),{ }^{13}$ leading to a better archival life time ${ }^{14}$ yet maintaining remarkable electronic and optical contrast, ${ }^{15}$ as required for application. However, the chemical stability of this composition was recently found to show a tendency toward phase separation ${ }^{16,17}$ into a Ge-rich and an $\mathrm{Sb}$-rich phase. The atomic structures of some $\mathrm{Ge}-\mathrm{Sb}$ compo-

${ }^{a)}$ Electronic mail: wuttig@physik.rwth-aachen.de. sitions have already been analyzed by Yoo et al. ${ }^{18}$ by means of x-ray diffraction (XRD) and extended x-ray absorption fine structure (EXAFS) at the Ge edge. They found evidence for $\mathrm{Ge}-\mathrm{Ge}$ bonds in crystallized $\mathrm{Ge}_{51} \mathrm{Sb}_{49}$ and also qualitatively in $\mathrm{Ge}_{13} \mathrm{Sb}_{87}$ but did not perform a quantitative analysis. Their measurements were performed at ambient temperature and therefore, the EXAFS data are strongly damped due to the large Debye-Waller factor. Dalba and Fornasini ${ }^{19}$ have demonstrated that lower temperatures significantly decrease the Debye-Waller factor for thin films of pure Ge. We have followed this idea and performed EXAFS $^{20}$ experiments at $10 \mathrm{~K}$, which led to a significant increase in data quality, i.e., a stronger amplitude especially at energies far above the edge. We will first present the experimental equipment, explain our data and finally discuss the results and conclude on the suitability of this compound in terms of PCM applications.

\section{METHODS}

Thin films of $\mathrm{Ge}_{15} \mathrm{Sb}_{85}$ were prepared by sputter deposition from stoichiometric targets on polymethyl methacrylate (PMMA) coated silica substrates. They were confirmed to be amorphous by means of XRD. The films were subsequently etched to remove the PMMA and scraped to powder. Some of the specimen was annealed for $30 \mathrm{~min}$ at $523(5) \mathrm{K}$ in a continuous flow of Argon, employing a heating rate of 5 $\mathrm{K} / \mathrm{min}$. The EXAFS measurements were performed on pellets, which were pressed from amorphous or crystallized powder, respectively, and were diluted in cellulose. 
Differential scanning calorimetry (DSC) was performed using a diamond DSC by Perkin Elmer with Ar purge gas and water cooling. The temperature scale was calibrated by the determination of the melting temperatures of indium and tin. Powder samples of $\mathrm{Ge}_{15} \mathrm{Sb}_{85}$ were thermally cycled in aluminum pans in order to determine transition temperatures and to make sure that the samples were contamination free. XRD patterns were recorded on sputter deposited films of approximately $0.25 \mu \mathrm{m}$ thickness on silicon substrates. The grazing incidence (GI) geometry was employed in a Phillips X'pert MRD with $\mathrm{Cu} K \alpha$ radiation.

EXAFS experiments were carried out at beamline $\mathrm{C}$ of DORIS III at HASYLAB. All data were obtained by absorption spectroscopy in transmission geometry at the $K$ edges of the two elements $\mathrm{Ge}$ and $\mathrm{Sb}$ at temperatures of approximately $10 \mathrm{~K}$. Two ionization chambers were used to monitor the beam intensity in front of and behind the sample. The first chamber was filled with $130 \mathrm{mbar} \mathrm{Ar}$ and the second with $880 \mathrm{mbar} \mathrm{Ar}$ for the Ge $K$ edge and 170 mbar and 1000 mbar of $\mathrm{Kr}$ for the $K$ edge of $\mathrm{Sb}$, respectively. At beamline $\mathrm{C}$, the $\mathrm{x}$-radiation passes a precollimator slit and is reflected in the direction of a double-crystal monochromator crystal set of $\mathrm{Si}(111)$ and $\mathrm{Si}(311)$ with the second crystal detuned in order to exclude higher order Bragg reflections. The incident beam has a cross section of approximately $2 \times 12 \mathrm{~mm}^{2}$. More details about the experimental setup ${ }^{21}$ and the monochromator employed ${ }^{22}$ have been published elsewhere.

Before evaluating structural information, the background from the obtained data was treated using the ATHENA software. ${ }^{23}$ The data analysis was performed by two different methods, the first of which relies on the ARTEMIS software, ${ }^{23}$ which is-like ATHENA-based on the algorithms of the IFEFFIT and FEFF (Ref. 24) software packages. The second method is a Reverse Monte Carlo (RMC) (Refs. 25 and 26) procedure, which also relies on the FEFF software. It has the additional benefit of generating three dimensional models of the structure, which are compatible with the bond lengths and numbers of bonds as obtained from EXAFS. The RMC code used for this study is custom made and starts from a highly distorted distribution of atoms to model the amorphous structure. Likewise, the algorithm starts from the crystalline structure of antimony for the crystalline data set with a value for the density as obtained by means of $\mathrm{x}$-ray reflection (XRR), i.e., $6.1(1) \mathrm{g} / \mathrm{cm}^{3}$ for the amorphous phase ${ }^{27}$ and $6.4(1) \mathrm{g} / \mathrm{cm}^{3}$ for the crystalline phase. Further details on the Monte Carlo calculations will be given in the next paragraph.

\section{RESULTS}

\section{A. Calorimetry and diffractometry}

The calorimetric analysis was performed on $5.04 \mathrm{mg}$ of $\mathrm{Ge}_{15} \mathrm{Sb}_{85}$ powder specimen, which were heated in a $20 \mu \mathrm{l} \mathrm{Al}$ crucible from ambient temperature to $773 \mathrm{~K}$ applying a heating rate of $5 \mathrm{~K} / \mathrm{min}$. Subsequently, the sample was cooled at $5 \mathrm{~K} / \mathrm{min}$ back to ambient temperature. The temperature program was repeated to verify that the peaks are not due to reversible phase transitions. The resulting scans-Fig. 1 -show two exothermal transitions upon first heating. Their

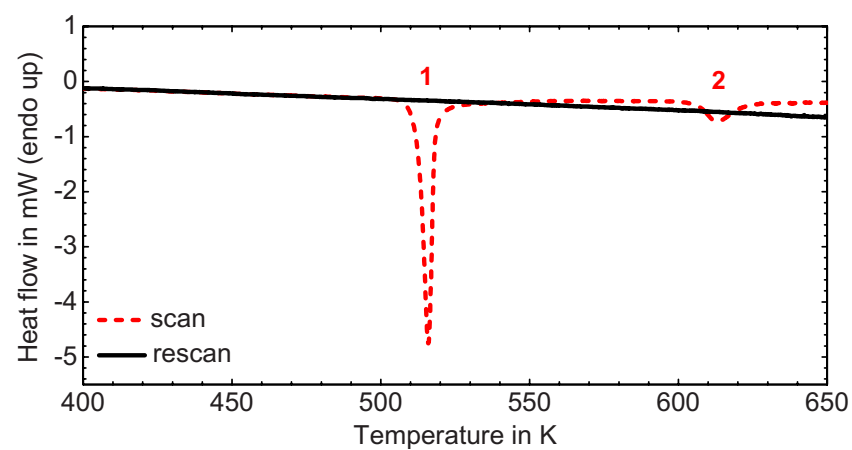

FIG. 1. (Color online) DSC temperature scan at a constant heating rate of $5 \mathrm{~K} / \mathrm{min}$ showing two peaks of crystallization, the first (1) of which is due to crystallization of an Sb-rich phase, while the second (2) is due to crystallization of a Ge-rich phase as confirmed by XRD (see Fig. 2). A slight drift of the zero-line is responsible for the difference between scan and rescan at higher temperatures.

onset temperatures are 512(1) and 606(1) $\mathrm{K}$ with the first transition having a significantly higher latent heat than the second.

Thin films of the same material were deposited on silica substrates and were heated to 523(5) and 653(5) $\mathrm{K}$ with holding times of $30 \mathrm{~min}$ and the same heating rate (i.e., $5 \mathrm{~K} / \mathrm{min}$ ). They were subsequently cooled and investigated by means of XRD. The first sample [523(5) K annealing temperature] shows a powder pattern (see Fig. 2) representing a rhombohedral symmetry $(\mathrm{R} \overline{\mathrm{3}} \mathrm{m})$ of the sample and lattice parameters $a=4.235 \AA$ and $c=11.341 \AA$ (i.e., $\left.a=4.502 \AA, \alpha=56.1^{\circ}\right)$, as is common for pure $\mathrm{Sb}(a$ $=4.308 \AA$ and $c=11.274 \AA \AA^{28}$ i.e., $a=4.506 \AA$ \& $\alpha=57.1^{\circ}$ ). The diffractogram of the specimen annealed at higher temperature [i.e., 653(5) K] shows reflections of the same rhombohedral geometry, yet slightly weaker rhombohedral distortions, $a=4.263 \AA$ and $c=11.192 \AA(a=4.469 \AA, \alpha$ $\left.=57.0^{\circ}\right)$. In the same diffractogram, some additional reflections appear which can be assigned to crystallites of pure Ge in the diamond structure. A separate evaluation of the full width at half maximum, according to Scherrer, ${ }^{29}$ allows for the determination of average sizes of coherent scattering do-

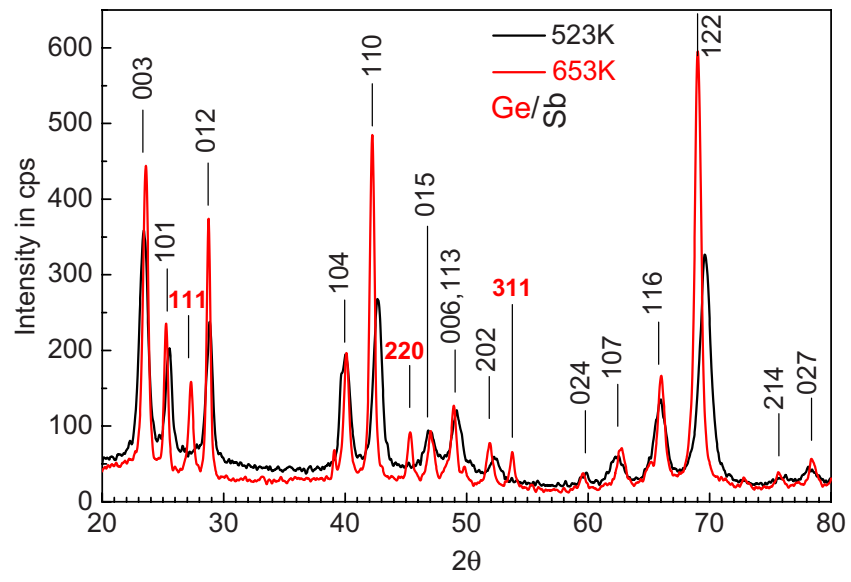

FIG. 2. (Color online) Diffractograms of $\mathrm{Ge}_{15} \mathrm{Sb}_{85}$ samples, which were crystallized at $523 \mathrm{~K}$ and $653 \mathrm{~K}$. Reflexes from rhombohedral $\mathrm{Sb}$ are indexed vertically, while reflexes of diamondlike Ge are indexed horizontally. The sample which was crystallized at $523 \mathrm{~K}$ does not show reflexes of diamondlike $\mathrm{Ge}$. The diffractograms were recorded using $\mathrm{Cu} K \alpha$ radiation. 


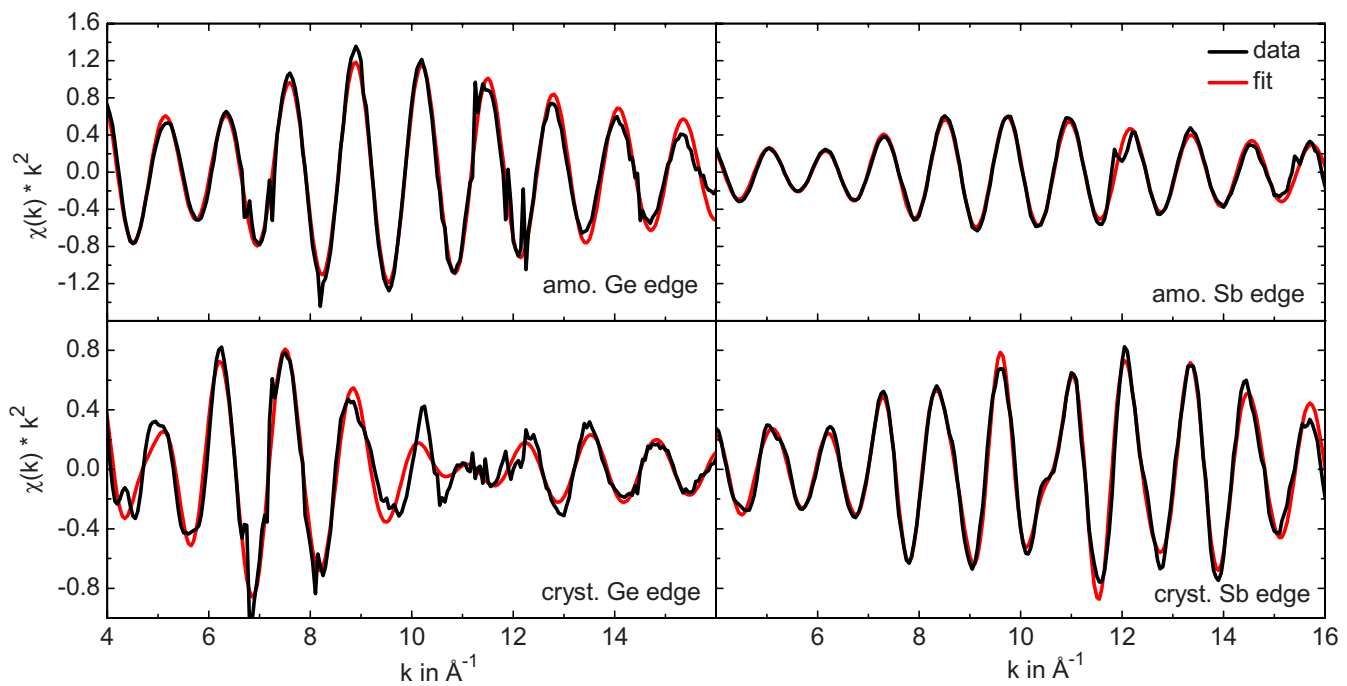

FIG. 3. (Color online) Top: EXAFS data for amorphous $\mathrm{Ge}_{15} \mathrm{Sb}_{85}$ with a fit using a model for a first coordination shell only. The fit reproduces all features of the data. Bottom: EXAFS data for crystallized $\mathrm{Ge}_{15} \mathrm{Sb}_{85}$ with a fit using two different models for each edge reproducing all features of the experimental data. The explanation in the text shows that this finding is indicative for phase separation.

mains. They turn out to be 17(2) $\mathrm{nm}$ for the Ge-rich phase and 15(1) $\mathrm{nm}$ for the Sb-rich phase. However, these reflections of Ge only appear after annealing at temperatures above 523(5) $\mathrm{K}$. This raises the question at which temperature the phase separation of $\mathrm{Ge}$ and $\mathrm{Sb}$ actually takes place. A quantitative analysis of the EXAFS data will answer this question in the following.

\section{B. EXAFS data treatment}

All EXAFS oscillations $\mu(E)$ were first normalized using the ATHENA software applying pre-edge subtraction and edge step normalization. The edge step $E_{0}$ was defined as the point of highest slope of the absorption over energy curve. Further edge energy corrections $(\Delta E)$ were applied relative to $E_{0}$ during the fitting process. All EXAFS spectra were recorded at least twice and subsequently averaged. The fine structure oscillations were extracted by subtracting all background effects, which are characterized by a spline function from 0 to $16.15 \AA^{-1}$ with the number of nodes given by 2 $\times R_{\mathrm{bkg}} \times \Delta k / \pi+1$. The parameter $R_{\mathrm{bkg}}$, i.e., the critical radius below which all oscillations are neglected, was defined as $1.3 \AA$ for all amorphous samples and $1.2 \AA$ for all crystalline samples.

\section{Qualitative discussion}

The EXAFS data for the $K$ edges of $\mathrm{Ge}$ and $\mathrm{Sb}$ are shown in $k$-space in Fig. 3. Oscillations are very strong in both phases up to high reciprocal wave vectors of $\sim 16 \AA^{-1}$, which is in part due to the weak damping, i.e., a small Debye-Waller factor at $\sim 10 \mathrm{~K}$. As expected, the crystalline data sets show additional beats, which originate from higher order coordination shells in the crystalline phase.

The Fourier transforms of the data have been calculated by applying window functions between 2 and $16 \AA^{-1}$. The first neighbor peaks in $r$-space (not shown here) are clearly visible and increase slightly in width after crystallization. This observation leads us to the conclusion that bond lengths in the crystallized sample are less well defined. The peak height at the Ge edge decreases significantly and gets broader upon crystallization. This shows that the short range ordering around Ge atoms is not well defined. This finding will be elaborated in more detail by a quantitative analysis in the following.

\section{Fitting scattering paths in a local model}

The EXAFS for the $\mathrm{Ge}$ and $\mathrm{Sb} K$ edges of the asdeposited sample are shown in Fig. 3 together with a fit to the data obtained by ARTEMIS. Both edges were fit independently due to the lack of a sufficiently large atomic model for the amorphous structure. Only the first shell of neighboring atoms of both species gives a significant signal in the EXAFS spectra. Therefore, this model allows for a determination of bond lengths and stoichiometries on the first coordination shell only.

Usually, the EXAFS signal is a sum of different scattering paths, which are best described by the single-scattering EXAFS formula ${ }^{20}$

$$
\begin{aligned}
\chi(k)= & S_{0}^{2}(k) \sum_{j} \frac{N_{j}}{k R_{j}^{2}} F_{j}(k) \cdot \exp \left(-2 k^{2} \sigma_{j}^{2}\right) \\
& \cdot \exp \left(-\frac{2 R_{j}}{\lambda}\right) \cdot \sin \left[2 k R_{j}+\delta_{j}(k)\right],
\end{aligned}
$$

with the sum running over all coordination shells, which have a certain distance from the absorbing atom. The given parameters and the fit procedures and results will be described in the following, beginning with the fit on the Ge edge of the as-deposited specimen. The most important parameters and the fitting results are summarized in Table I.

The model for the Ge edge consists of two scattering paths (Ge and $\mathrm{Sb}$ backscatterers) with individual radii $R_{j}$ and coordination numbers $N_{j}$ multiplied to the passive electron reduction factors $S_{0}^{2}$ and one Debye-Waller factor $\sigma_{\mathrm{Ge}}$. The FEFF software ${ }^{24}$ was used to calculate the phase shift $\delta_{j}(k)$, the scattering amplitude $F_{j}(k)$ and the mean free 
TABLE I. Amorphous $\mathrm{Ge}_{15} \mathrm{Sb}_{85}$ : scattering paths that were used at both edges. The absorbing atom (edge) is named first, followed by the backscattering species. The partial numbers of neighbors $N_{j}$ of $\mathrm{Ge}$ and $\mathrm{Sb}$ reproduce the stoichiometry of the sample.

\begin{tabular}{lcccc}
\hline \hline Path: Abs.-Scat. & $N_{j}$ & $\begin{array}{c}R_{j} \\
(\AA)\end{array}$ & $\begin{array}{c}\sigma^{2} \\
\left(\AA^{2}\right)\end{array}$ & $\begin{array}{c}\Delta E \\
(\mathrm{eV})\end{array}$ \\
\hline $\mathrm{Ge}-\mathrm{Sb}$ & $3.4(3)$ & $2.66(1)$ & $0.0025(5)$ & $+3.2(2)$ \\
$\mathrm{Ge}-\mathrm{Ge}$ & $0.6(2)$ & $2.46(2)$ & $0.0025(5)$ & $+3.2(2)$ \\
$\mathrm{Sb}-\mathrm{Ge}$ & $0.6(1)$ & $2,67(1)$ & $0.0022(3)$ & $+7.4(4)$ \\
$\mathrm{Sb}-\mathrm{Sb}$ & $2.6(2)$ & $2.87(1)$ & $0.0022(3)$ & $+7.4(4)$ \\
\hline \hline
\end{tabular}

path of the photoelectron $\lambda$. This model was refined by adjusting all remaining parameters until the best agreement between data and calculation was obtained. A single energy shift $\Delta E_{\mathrm{Ge}}=+3.2(2) \mathrm{eV}$ was determined for both scattering paths during a fit in $r$-space from 1.6 to $3.9 \AA$. The fit resulted in a residual ${ }^{30}$ of 0.013 . Concentration factors were fitted by the relative amplitudes of $N_{1} \mathrm{Sb}$ and $N_{2}$ Ge backscattering atoms, leading to a local stoichiometry of $\mathrm{Ge}_{15(3)} \mathrm{Sb}_{85(3)}$ around Ge. The $S_{0}^{2}$ factor is 0.80(5), if the number of nearest neighbors $N_{1}+N_{2}=4$ on the first coordination shell is assumed.

The model for the $S b$ edge has been set up like that at the $\mathrm{Ge}$ edge with a single Debye-Waller factor $\sigma_{\mathrm{Sb}}=0.0022(3) \AA^{2}$ and an energy shift $\Delta E_{\mathrm{Sb}}=+7.4(4) \mathrm{eV}$. The fit has been performed in $r$-space with a window function between 1.7 and $3.4 \AA$ resulting in a residual of 0.009 . The local stoichiometry around $\mathrm{Sb}$ turns out to be $\mathrm{Ge}_{18(3)} \mathrm{Sb}_{82(3)}$ with a passive electron reduction factor of $0.88(5)$, if the number of nearest neighbors $(\mathrm{Ge}+\mathrm{Sb}), N_{1}$ $+N_{2}=3$, is assumed.

Both local models each contain a total number of 6 independent free parameters, which is smaller than that resulting from the Nyquist criterion, 20. The fact that a good fit could be obtained with significantly less free parameters is indicative for the suitability of the model. Assuming the passive electron reduction factors $S_{0}^{2}$ determined for the crystalline state $[0.83(8)$ for $\mathrm{Sb}$ and $0.8(1)$ for $\mathrm{Ge}]$ to be correct in the amorphous samples as well-an assumption that is commonly made-it turns out that Ge atoms have 4.0 neighboring atoms, while $\mathrm{Sb}$ atoms have 3.2 neighbors. Therefore, the results agree with the local application of the 8-N rule, predicting fourfold coordinated $\mathrm{Ge}$ and threefold coordinated $\mathrm{Sb}$. At the Ge edge, average bond lengths of 2.46(2) $\AA$ for $\mathrm{Ge}-\mathrm{Ge}$ bonds and 2.66(1) $\AA$ for $\mathrm{Ge}-\mathrm{Sb}$ bonds are found. On the $\mathrm{Sb}$ edge, $\mathrm{Sb}-\mathrm{Ge}$ distances are 2.67(1) $\AA$ with $\mathrm{Sb}-\mathrm{Sb}$ distances of 2.87(1) $\AA$. The Ge-Sb bond lengths are consistent and indicate that amorphous $\mathrm{Ge}_{15} \mathrm{Sb}_{85}$ consists of a single phase.

The EXAFS data for the crystallized sample are shown in Fig. 3 together with a fit by means of ARTEMIS. A description of the model employed to fit each edge will be given, beginning with the model for the Sb edge. Due to the large relative concentration of backscattering $\mathrm{Sb}$ atoms, this model starts from the rhombohedral structure of crystalline pure $\mathrm{Sb}$ with a certain fraction of $\mathrm{Sb}$ atoms replaced by $\mathrm{Ge}$ on each coordination shell around the absorbing atom. A determination of the local stoichiometry around $\mathrm{Sb}$ atoms is possible
TABLE II. Crystallized sample of $\mathrm{Ge}_{15} \mathrm{Sb}_{85}$, Sb edge. $S_{0}^{2}=0.83(8)$. The occupation of the first shell with Ge atoms is reduced as compared to the stoichiometry in the amorphous samples. On shell no. 1, Ge atoms are displaced toward the absorbing atom and on shell no. 2, they are displaced away from the absorber, i.e., toward the next $\mathrm{Sb}$ atom. Values denoted with a star were expressed by the lattice constants $u, a$, and $\alpha$ during the fitting procedure.

\begin{tabular}{lllll}
\hline \hline Path: Abs.-Scat. & $N_{j}$ & \multicolumn{1}{c}{$\begin{array}{c}R_{j} \\
\AA\end{array}$} & $\begin{array}{c}\sigma_{j}^{2} \\
\left(\AA^{2}\right)\end{array}$ & $\begin{array}{c}\Delta E \\
(\mathrm{eV})\end{array}$ \\
\hline Sb-Sb no. 1 & 2.7 & $2.88^{*}$ & $0.0017(4)$ & +7.2 \\
Sb-Ge no. 1 & 0.3 & $2.74(2)$ & $0.0017(4)$ & +7.2 \\
Sb-Sb no. 2 & 2.7 & $3.34^{*}$ & $0.007(1)$ & +7.2 \\
Sb-Ge no. 2 & 0.3 & $3.5(2)$ & $0.007(1)$ & +7.2 \\
Sb-Sb no. 3 & 5.4 & $4.36^{*}$ & $0.007(1)$ & +7.2 \\
Sb-Ge no. 3 & 0.6 & $4.2(2)$ & $0.007(1)$ & +7.2 \\
Sb-Sb no. 4 & 5.4 & $4.52^{*}$ & $0.007(1)$ & +7.2 \\
Sb-Ge no. 4 & 0.6 & $3.83(9)$ & $0.007(1)$ & +7.2 \\
\hline \hline
\end{tabular}

by introducing a factor for the Ge/Sb ratio on every coordination shell. This model can also be used to determine the lattice constants of $\mathrm{Sb}$ (i.e., $a, u$, and $\alpha$ ) as well as the bond lengths $R_{j}$ of $\mathrm{Ge}$ atoms. A complete list of all considered scattering paths can be found in Table II. They are all based on a single free parameter for the energy shift $\Delta E_{\mathrm{Sb}}$. DebyeWaller factors were introduced and fitted for the first coordination shell $\sigma_{1}$ and for all higher coordination shells $\sigma_{2}$. All bond lengths $R_{j}$ of $\mathrm{Sb}$ atoms are defined as functions of the three lattice parameters $a, u$, and $\alpha$. The resulting values are $a=4.52(2) \AA, \alpha=56.0(3)^{\circ}$, and $u=0.233(1)$, indicating a slightly stronger distortion of the unit cell angle ${ }^{31}$ than that found for single crystals of Sb: $a=4.507 \AA, \alpha=57.1^{\circ}$, and $u=0.233$. $^{28}$

The EXAFS spectrum for the $S b$ edge can be reproduced with a residual of 0.029 by a model with a local stoichiometry of $\mathrm{Ge}_{10(3)} \mathrm{Sb}_{90(3)}$, i.e., with a slightly lower relative content of Ge than in the macroscopic sample. The short range order in pure $\mathrm{Sb}$ is determined by the rhombohedrally distorted simple cubic geometry with three neighboring atoms at $2.88 \AA$ and three atoms at $3.34 \AA$. In this structure, $\mathrm{Ge}$ atoms on the first coordination shell at $2.88 \AA$ tend to be dislocated toward the closest $\mathrm{Sb}$ atom, leading to a decrease in the bond length on the first shell of -0.14(2) $\AA$ and to an increase on the second shell by $+0.2(1) \AA$, which corresponds to a shortening of the bond to the next $\mathrm{Sb}$ atom along the rhombohedral 111 direction. Interestingly, those shifts are consistent (they should ideally have the same modulus with opposite sign) and tell us that Ge atoms are displaced from the ideal lattice sites of elemental Sb.

Based on the reduced concentration of $\mathrm{Ge}$ around $\mathrm{Sb}$, one can suspect the existence of two phases, which appear superimposed in the EXAFS data: one in which Ge atoms are located on an $\mathrm{Sb}$ lattice site and another with higher concentrations of Ge. This assumption will be further supported by the evaluation at the Ge edge. Assuming a fixed number of 3 neighboring atoms, the passive electron reduction factor $S_{0}^{2}$ turns out to be $0.83(8)$. The presented model has been fit in real space between 1.7 and $4.8 \AA$, thus allowing for 26 independent variables (Nyquist criterion), only 12 
TABLE III. Crystallized sample of $\mathrm{Ge}_{15} \mathrm{Sb}_{85}$, Ge edge. $S_{0}^{2}=0.8(1)$. At 2.43(1) $\AA$ bond length, there is a high amount of Ge backscatterers (I), which can be explained by phase separation. Phase (I) consists of fourfold coordinated Ge, while phase (II) is a crystal of antimony with some Ge atoms on each coordination shell.

\begin{tabular}{lclll}
\hline \hline Path: Abs.-Scat. & Contrib. & $\begin{array}{c}R_{j} \\
(\AA)\end{array}$ & $\begin{array}{c}\sigma^{2} \\
\left(\AA^{2}\right)\end{array}$ & $\begin{array}{c}\Delta E \\
(\mathrm{eV})\end{array}$ \\
\hline Ge-Ge (I) & $0.37(5)$ & $2.43(1)$ & $0.004(1)$ & +5.2 \\
Ge-Sb (II) & $0.63(5)$ & $2.73(2)$ & $0.004(1)$ & +5.2 \\
Ge-Ge (II) & $0.63(5)$ & $2.79(8)$ & $0.004(1)$ & +5.2 \\
Ge-Sb (II) & $0.63(5)$ & $3.23(3)$ & $0.02(1)$ & +5.2 \\
Ge-Ge (II) & $0.63(5)$ & $3.3(7)$ & $0.02(1)$ & +5.2 \\
\hline \hline
\end{tabular}

of which were necessary in the fit. The low resulting residual $R$ of 0.029 together with the low number of free parameters shows that the fit is well controlled.

The EXAFS data on the Ge edge of the crystallized sample are shown in Fig. 3. The model presented earlier, i.e., a rhombohedral structure of $\mathrm{Sb}$, cannot reproduce all features at this edge. Therefore, the fit presented in the following is based on a superposition of two phases but has to be restricted to the first and second coordination shells of the data because contributions from higher shells are too weak to allow for a further evaluation of scattering paths. The first phase (I) is the same as that presented above for the Sb edge and the second phase (II) contributing to the model is bulk fourfold coordinated Ge. The second model does not allow for discriminating amorphous and crystalline Ge due to the fact that the short range ordering is very similar in both structures. Nevertheless, we see in the x-ray diffractogram that no visible reflections of crystalline Ge exist after this annealing procedure and, therefore, it is likely that Ge occurs as an amorphous phase, which crystallizes at higher temperatures, as shown in Fig. 1. This way of fitting allows for determining the relative contributions of both local structures. It turns out that the Sb-rich structure (I) contributes $63(5) \%$, while the crystalline Ge structure (II) contributes $37(5) \%$. One Debye-Waller factor was employed for the first coordination shell in both structures and was determined to be $\sigma_{1}=0.004(1) \AA^{2}$ after the fit. The DebyeWaller factor for the second shell [refined only in (II)] is $\sigma_{2}=0.016(13) \AA^{2}$.

Higher order cumulants, i.e., anharmonic contributions to the Debye-Waller factors, have not been taken into account throughout this analysis, because on the one hand the harmonic approximation already leads to a very accurate fit and on the other hand because anharmonic effects are expected to be negligible at low temperatures of $\sim 10 \mathrm{~K}$.

Table III shows all scattering paths that were taken into account to fit the data. The superposition of two phases and the contribution of a phase with accumulated atoms of $\mathrm{Ge}$ are key findings for this specimen.

Therefore, the evaluation shows clear indications of phase separation. This trend can also be extracted from the data using a different approach, delivering not only a model for the short range order. RMC was employed to create a structural model for the long range order, which is compatible with the EXAFS data.

\section{E. Fitting with RMC}

A RMC simulation delivers a three dimensional model for the atomic structure of the investigated sample by fitting several data sets simultaneously. In this study, the EXAFS data of $\mathrm{Ge}$ and $\mathrm{Sb} K$ edges are used at the same time. Starting from a configuration of 864 atoms on a lattice of crystalline antimony, the FEFF software is used to calculate all $k$-dependent functions of the EXAFS formula (1). Several other values, for which no commonly employed $a b$ initio theory exists yet, were set to the values determined by ARTEMIS (see above). These are the Debye-Waller factors $\sigma^{2}$, the edge corrections $\Delta E$ and the passive electron reduction factors $S_{0}^{2}$. With these prerequisites at hand, the EXAFS formula (1) allows for calculating the scattering paths for each configuration of atoms.

The RMC algorithm tries to randomly displace or swap atoms in each iteration step and recalculates the EXAFS according to formula (1). The deviation from the experimental data is defined as

$$
\chi^{2}=\sum_{k}\left[k^{2} \chi_{\exp }(k)-k^{2} \chi_{\text {calc }}(k)\right]^{2}
$$

thereby weighting the EXAFS data by $k^{2}$. If the discrepancy decreases, the displacement is accepted and if it increases, it is either accepted or rejected depending on a virtual temperature parameter, which is continuously reduced during the fit. This method is similar to the well known Metropolis Monte Carlo method with the total energy replaced by the $\chi^{2}$ of the fit. Two constraints are fixed within the code in order to compensate for the lack of an atomic potential in RMC: one limits the maximum displacement from the initial position, while the other employs hard sphere repulsive potentials around all atoms, thus limiting the minimum distance between neighboring atoms. Those constraints were checked not to limit the displacements of any sort of atoms, i.e., no "piling" of bonds at the constraints occurs.

The RMC technique is based on an intrinsically large number of highly constrained atomic coordinates which are randomly adjusted in order to reproduce the original atomic structure as closely as possible. Therefore, one has to keep in mind that the final result is not unique but can be regarded as an indicator for trends in further analyses.

The initial configuration for the data on as-deposited $\mathrm{Ge}_{15} \mathrm{Sb}_{85}$ is based on a box containing 864 atoms. $15 \%$ of the atoms were defined to be $\mathrm{Ge}$ in order to get the correct macroscopic composition. The size of the box was chosen to result in a mass density of $6.1(1) \mathrm{g} / \mathrm{cm}^{3}$ as determined by $\mathrm{XRR}^{27}$ This structure was used to calculate all EXAFS single scattering paths by FEFF with fixed Debye-Waller factors of $0.002 \AA^{2}$. All scattering paths were shifted in $k$-space with fixed energy shifts $\Delta E$ of +5.2 and $+7.2 \mathrm{eV}$ as determined above for the crystalline structure at the $\mathrm{Ge}$ and $\mathrm{Sb}$ edge, respectively. The scattering paths in $k$-space were summed according to the standard EXAFS formula for single scattering paths (1). Multiple scattering paths were not taken into account, because they have amplitudes below 5\% according to FEFF. $S_{0}^{2}$ for Ge and $\mathrm{Sb}$ were fixed to the previously determined values of 0.80 and 0.83 , respectively. 


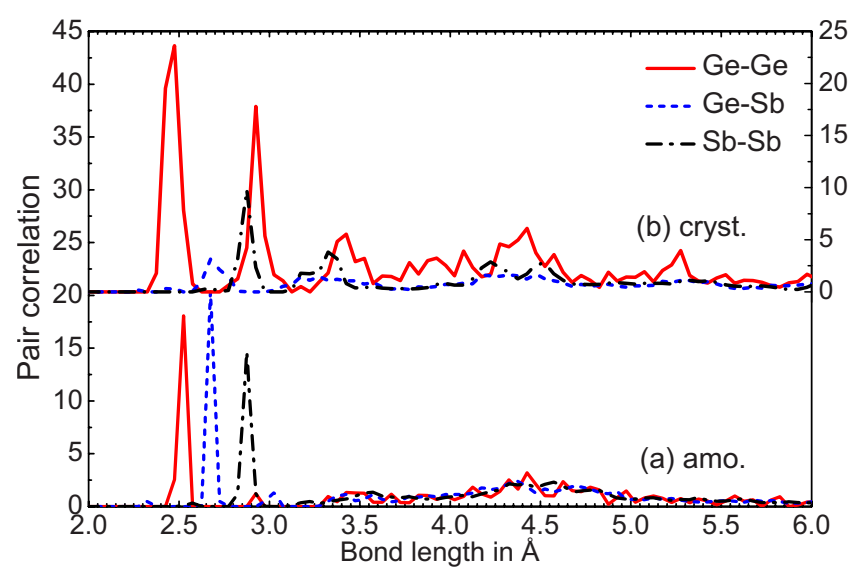

FIG. 4. (Color online) Relative RDF resulting from RMC runs on amorphous and crystalline $\mathrm{Ge}_{15} \mathrm{Sb}_{85}$. In the amorphous phase (lower curve) only one bond length is visible for $\mathrm{Sb}-\mathrm{Sb}$. The $\mathrm{Ge}-\mathrm{Ge}$ distribution function shows a significant peak which originates from short covalent $\mathrm{Ge}-\mathrm{Ge}$ bonds. Additionally, the large peak of Ge-Sb bonds as compared to the crystallized sample shows that the Ge atoms are randomly mixed within the network of $\mathrm{Sb}$. Crystallized $\mathrm{Ge}_{15} \mathrm{Sb}_{85}$ (upper curve): two $\mathrm{Sb}-\mathrm{Sb}$ bond lengths are visible, as expected for crystalline Sb. Several Ge-Ge bonds exist with different bond lengths, but the amount of Ge-Sb bonds is very low, thus indicating that the interface area between $\mathrm{Ge}$ and $\mathrm{Sb}$ is reduced as compared to the as-deposited sample. This effect can be explained by phase separation into an Sb-rich and a Ge-rich phase.

\section{F. Results from RMC}

The result of the RMC run, i.e., a set of coordinates of 864 atoms, was analyzed by calculating the radial distribution function (RDF), which is shown in Fig. 4(a) for the as-deposited amorphous sample. Only peaks from nearest neighbors are visible, while at radii above $3 \AA$ the distribution is flat and featureless. This is a consequence of the absence of long-range order in amorphous materials. Figure 4(a) shows only one significant $\mathrm{Sb}-\mathrm{Sb}$ bond length, while two peaks are observed for Ge-Ge bonds around 2.5 and $2.9 \AA$ with well defined, i.e., narrow peaks. The first one is more intense and shows that whenever Ge-Ge bonds are present, they have a bond length of $2.45 \AA$, which is common for elemental Ge. ${ }^{32}$ A strong peak exists for $\mathrm{Ge}-\mathrm{Sb}$ bonds at $2.7 \AA$, which is characteristic for the formation of an amorphous compound of $\mathrm{Ge}$ and $\mathrm{Sb}$. Indeed, the three dimensional model (not shown here) shows that there is no accumulation of Ge atoms. For the crystallized sample, however, this peak is drastically decreased in intensity, providing another indication of phase separation of $\mathrm{Ge}$ and $\mathrm{Sb}$, as discussed next.

The result of an RMC run on the data sets of the crystallized sample can be seen in the RDF in Figs. 4(b) and 5(b), showing a box of 864 atoms. The stronger features in the RDF above $3 \AA$ as compared to Fig. 4(a) clearly prove the crystallinity of the sample. The RDF shows two peaks for $\mathrm{Sb}-\mathrm{Sb}$ bonds at 2.8 and $3.3 \AA$. These two values match the data for crystalline $\mathrm{Sb}$, i.e., 2.91 and $3.36 \AA^{33}$ At the same time, the number of $\mathrm{Ge}-\mathrm{Ge}$ bonds increases and $\mathrm{Ge}-\mathrm{Sb}$ bonds around $2.7 \AA$ almost disappear as compared to the RDF of amorphous $\mathrm{Ge}_{15} \mathrm{Sb}_{85}$ [see Fig. 4(a)]. Both differences again originate from the phase separation in the crystallized sample.
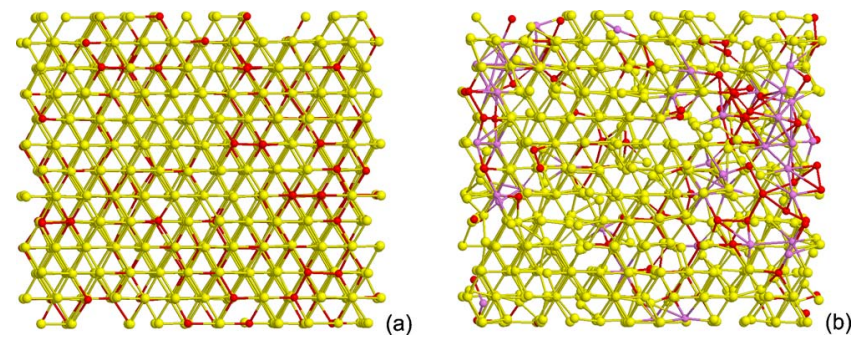

FIG. 5. (Color online) The initial (left) and final (right) box of 864 atoms of $\mathrm{Ge}_{15} \mathrm{Sb}_{85}$ for an RMC calculation. $\mathrm{Sb}$ atoms are shown in light/yellow color and $\mathrm{Ge}$ atoms in dark/red or purple color depending on their bond lengths. The atom is shown in purple if at least one bond is shorter than $2.65 \AA$. A significant clustering of Ge atoms can be observed (periodic boundary conditions were employed).

The boxes of atoms-Figs. 5(a) and 5(b) - show Sb atoms in yellow, while Ge atoms with long bond lengths $(>2.65 \AA)$ are colored red and Ge atoms with short bond length $(<2.65 \AA)$ are shown in blue. The short bonds are characteristic for elemental Ge (purple), while the longer bonds are characteristic for Ge atoms (red), which are in a distorted octahedral environment. ${ }^{32,34}$ Starting from an initial structure of randomly mixed atoms, phase separation hence occurs by agglomeration of $\mathrm{Ge}$. The contribution of $\mathrm{Sb}$ at the Ge edge found in the ARTEMIS evaluation can be explained by $\mathrm{Ge}-\mathrm{Sb}$ interfaces. Hence, the results of the two different methods (path fitting and RMC) are consistent.

\section{CONCLUSIONS AND DISCUSSION}

The bonding in as-deposited amorphous $\mathrm{Ge}_{15} \mathrm{Sb}_{85}$ has pronounced covalent character. This can be seen by the locally fulfilled $8-\mathrm{N}$ rule with four nearest neighbors around $\mathrm{Ge}$ and three around $\mathrm{Sb}$. The corresponding experimental values are 4.0 and 3.2. Bond lengths in amorphous $\mathrm{Ge}_{15} \mathrm{Sb}_{85}$ were determined to be 2.46(2), 2.67(1), and 2.87(1) $\AA$ for $\mathrm{Ge}-\mathrm{Ge}, \mathrm{Ge}-\mathrm{Sb}$, and $\mathrm{Sb}-\mathrm{Sb}$ bonds, respectively. These values agree within the error bars with those observed in the common phase-change material $\mathrm{Ge}_{2} \mathrm{Sb}_{2} \mathrm{Te}_{5}: 2.48$ and $2.69 \AA$ for $\mathrm{Ge}-\mathrm{Ge}$ and $\mathrm{Ge}-\mathrm{Sb}$ bonds, respectively. At the same time, the Debye-Waller factors are small and the stoichiometry is consistent for the fits at both absorption edges. However, the RMC runs in Fig. 4 show a second Ge-Ge bond length around $2.9 \AA$, which is not a necessary contribution when fitting the data with ARTEMIS. We, therefore, assume that the $\mathrm{RDF}$ of these bonds is too small and too widely spread to be observed in the EXAFS data. Therefore, the peak in Fig. 4 originates from the necessity in RMC to create a consistent three dimensional structural model.

In the crystallized sample, the absorption spectra look significantly different from those of the amorphous specimen. Additionally, the concentration of Ge atoms on the shell of nearest neighbors deviates at the two edges. The most suitable model to fit the data of the crystallized sample is that of a superposition of two phases, i.e., (I) fourfold coordinated Ge atoms and (II) Ge atoms located on sites in a crystal of pure antimony. In phase (II) the Ge atoms do not remain on their lattice sites, but rather shift, thus decreasing the short bond length by $0.18(2)$ to $2.73(2) \AA$. The fourfold coordinated Ge (I) is considered to be in an amorphous struc- 
ture immediately after crystallization of the Sb-rich phase. This was shown by the complementary powder diffraction patterns, where reflections of pure Ge evolve and background intensity decreases at higher temperatures than the annealing temperature and the fact that we observe a second transition at $606(1) \mathrm{K}$ in the calorimetric data. It is worth mentioning that the $\mathrm{Ge}-\mathrm{Ge}$ bond length in the crystallized sample $[2.43(1) \AA]$ turns out to be slightly shorter than that of the as-deposited amorphous sample [2.46(2) $\AA$ ]. This deviation might originate from the higher local concentration of $\mathrm{Ge}-\mathrm{Ge}$ bonds per Ge atom in the crystallized sample. shortening also the $\mathrm{Ge}-\mathrm{Sb}$ bonds as compared to the amorphous sample, where the local concentration of $\mathrm{Ge}$ is lower.

All observations are consistent with calorimetric ${ }^{16}$ and diffraction ${ }^{17}$ data from the literature. The second transition has a well defined onset temperature and therefore cannot be due to a continuous agglomeration of $\mathrm{Ge}$ atoms. We have shown that the phase separation already takes place at temperatures significantly below $606 \mathrm{~K}$, forming regions with high concentrations of amorphous $\mathrm{Ge}$, which then crystallize around $606 \mathrm{~K}$.

It has been shown that phase separation takes place during annealing at about 523(5) $\mathrm{K}$ on the time scale of minutes. In phase change memory devices, the active medium is heated and thus crystallized on the time scale of nanoseconds. However, the longer time scales are relevant for the archival life time of the data. Additionally, it is important for the investigation of these effects which are responsible for the large optical (i.e., resonance bonding ${ }^{35}$ ) and electronic contrast, where usually longer annealed samples are investigated. Therefore, phase separation has to be taken into account when investigating oven-annealed $\mathrm{Ge}_{15} \mathrm{Sb}_{85}$.

In phase change memory devices, the material should commonly be reset by melting the eutectic at $903 \mathrm{~K}$ to a single liquid phase. But the melting temperature of $\mathrm{Ge}$ enriched regions with a higher concentration of Ge (e.g., $50 \%)$ is significantly higher. It seems important to heat significantly above $903 \mathrm{~K}$ to melt the active material completely, because otherwise clusters of segregated $\mathrm{Ge}_{\mathrm{x}} \mathrm{Sb}_{1-\mathrm{x}}$ with $\mathrm{x}<15 \%$ might remain at the cold electrodes, thus modifying the local stoichiometry. Therefore, it is crucial to ensure a homogeneous temperature distribution throughout the active material. As the most important reason for the introduction of $\mathrm{Ge}$ to $\mathrm{Sb}$ is to increase the amorphous stability, one might wonder if heavier dopants like Sn can also stabilize the amorphous structure, without sacrificing the stability against phase separation.

\section{ACKNOWLEDGMENTS}

The authors would like to thank the Deutsche Forschungsgemeinschaft for support under Grant Nos. Wu 243/12 and BE 1653/18, as well as for travel support by PROCOPE and P. Merkelbach for the preparation of samples. Portions of this research were carried out at the light source facilities DORIS III at HASYLAB/DESY. DESY is a member of the Helmholtz Association (HGF). We would like to thank E. Welter for providing valuable help at beamline $\mathrm{C}$ at HASYLAB and $\mathrm{M}$. Woda for the XRR measurements on thin films of $\mathrm{Ge}_{15} \mathrm{Sb}_{85}$.

${ }^{1}$ M. Wuttig and N. Yamada, Nature Mater. 6, 824 (2007).

${ }^{2}$ S. R. Ovshinsky, Phys. Rev. Lett. 21, 1450 (1968).

${ }^{3}$ F. Bedeschi, R. Fackenthal, C. Resta, E. M. Donze, M. Jagasivamani, E. C. Buda, F. Pellizzer, D. W. Chow, A. Cabrini, G. Calvi, R. Faravelli, A. Fantini, G. Torelli, D. Mills, R. Gastaldi, G. Casagrande, and Numonyx, IEEE J. Solid-State Circuits 44, 217 (2009).

${ }^{4}$ G. Bruns, P. Merkelbach, C. Schlockermann, M. Salinga, M. Wuttig, T. D. Happ, J. B. Philipp, and M. Kund, Appl. Phys. Lett. 95, 043108 (2009).

${ }^{5}$ I. Friedrich, V. Weidenhof, W. Njoroge, P. Franz, and M. Wuttig, J. Appl. Phys. 87, 4130 (2000).

${ }^{6}$ J. Solis and C. N. Afonso, Appl. Phys. A: Mater. Sci. Process. 76, 331 (2003).

${ }^{7}$ Y. C. Chen, C. T. Rettner, S. Raoux, G. W. Burr, S. H. Chen, R. M. Shelby, M. Salinga, W. P. Risk, T. D. Happ, G. M. McClelland, M. Breitwisch, A. Schrott, J. B. Phillipp, M. H. Lee, R. Cheek, T. Nirschl, M. Lamorey, C. F. Chen, E. Joseph, S. Saidi, B. Yee, H. L. Lung, R. Bergmann, and C. Lam, Tech. Dig. - Int. Electron Devices Meet. 2006, 1.

${ }^{8}$ D. Lencer, M. Salinga, B. Grabowski, T. Hickel, J. Neugebauer, and M. Wuttig, Nature Mater. 7, 972 (2008).

${ }^{9}$ J. Siegel, C. N. Afonso, and J. Solis, Appl. Phys. Lett. 75, 3102 (1999).

${ }^{10}$ C. E. Wickersham, G. Bajor, and J. E. Greene, Solid State Commun. 27, 17 (1978).

${ }^{11}$ L. van Pieterson, M. H. R. Lankhorst, M. van Schijndel, A. E. T. Kuiper, and J. H. J. Roosen, J. Appl. Phys. 97, 083520 (2005).

${ }^{12}$ A. K. Petford-Long, R. C. Doole, C. N. Afonso, and J. Solis, J. Appl. Phys. 77, 607 (1995).

${ }^{13}$ J. A. Kalb, M. Wuttig, and F. Spaepen, J. Mater. Res. 22, 748 (2007).

${ }^{14}$ L. van Pieterson, J. C. N. Rijpers, and J. Hellmig, Jpn. J. Appl. Phys., Part 1 43, 4974 (2004).

${ }^{15}$ S. M. Wiggins, J. Bonse, J. Solis, C. N. Afonso, K. Sokolowski-Tinten, V. V. Temnov, P. Zhou, and D. von der Linde, J. Appl. Phys. 98, 113518 (2005).

${ }^{16}$ C. Cabral, L. Krusin-Elbaum, J. Bruley, S. Raoux, V. Deline, A. Madan, and T. Pinto, Appl. Phys. Lett. 93, 071906 (2008).

${ }^{17}$ S. Raoux, C. Cabral, L. Krusin-Elbaum, J. L. Jordan-Sweet, K. Virwani, M. Hitzbleck, M. Salinga, A. Madan, and T. L. Pinto, J. Appl. Phys. 105, 064918 (2009).

${ }^{18}$ Y. G. Yoo, D. S. Yang, H. J. Ryu, W. S. Cheong, and M. C. Baek, Mat. Sci. Eng., A 449-451, 627 (2007).

${ }^{19}$ G. Dalba and P. Fornasini, J. Synchrotron Radiat. 4, 243 (1997).

${ }^{20}$ D. C. Koningsberger and R. Prins, X-Ray Absorption (Wiley, New York, 1988).

${ }^{21}$ K. Rickers, W. Drube, H. Schulte-Schrepping, E. Welter, U. Brüggmann, M. Herrmann, J. Heuer, and H. Schulz-Ritter, AIP Conf. Proc. 882, 905 (2007).

${ }^{22}$ K. Rickers, U. Brüggmann, W. Drube, M. Herrmann, J. Heuer, E. Welter, H. Schulte-Schrepping, and H. Schulz-Ritter, AIP Conf. Proc. 879, 907 (2007).

${ }^{23}$ B. Ravel and M. Newville, J. Synchrotron Radiat. 12, 537 (2005).

${ }^{24}$ M. Newville, J. Synchrotron Radiat. 8, 96 (2001)

${ }^{25}$ R. L. McGreevy and L. Pusztai, Mol. Simul. 1, 359 (1988).

${ }^{26}$ R. L. McGreevy J. Phys.: Condens. Matter 13, R877 (2001).

${ }^{27} \mathrm{M}$. Woda, private communication (2008).

${ }^{28}$ R. W. G. Wyckoff, Crystal Structures (Krieger, Malabar, 1982).

${ }^{29}$ P. Scherrer, Nachr. Ges. Wiss. Goettingen, Math.-Phys. Kl. 26, 98 (1918).

${ }^{30}$ The residual $\mathrm{R}$ in this context is an indication of the fit's quality, with a good fit having a low residual. It is defined by: $R=\Sigma_{k}\left[k^{n} \chi_{\exp }(k)\right.$ $\left.-k^{n} \chi_{\text {calc }}(k)\right]^{2} / \Sigma_{k} k^{n} \chi_{\text {exp }}(k)^{2}$ with the exponent $n=2$, if not stated otherwise.

${ }^{31}$ At this point it seems important to explain, why it is possible to derive angular values from EXAFS, which itself is not sensitive to bond angles in the single scattering approximation: the crystalline structure of $\mathrm{Sb}$ is well known and has been checked by means of XRD to be correct for $\mathrm{Ge}_{15} \mathrm{Sb}_{85}$ as well. Therefore, the angular lattice constant $\alpha$ can be derived from the relative bond lengths of subsequent coordination shells.

${ }^{32}$ T. Hom, W. Kiszenick, and B. Post, J. Appl. Crystallogr. 8, 457 (1975). 
${ }^{33}$ N. C. Norman, Chemistry of Arsenic, Antimony and Bismuth (Blackie Academic \& Professional, London, 1998), p. 16.

${ }^{34}$ However, the distribution of bonds among the atoms is not contained in the EXAFS data and the coloring of atoms is undertaken here solely to show that these bond lengths exist in the model and that they preferably occur between two Ge atoms, i.e. in the Ge-enriched regions.

${ }^{35}$ K. Shportko, S. Kremers, M. Woda, D. Lencer, J. Robertson, and M. Wuttig, Nature Mater. 7, 653 (2008). 